\title{
Plunging jets from orifices of different geometry
}

\author{
Giorgio Moscato*, Giovanni Paolo Romano* \\ University La Sapienza, Dept. Mechanical \& Aerospace Engineering, Rome, Italy \\ *giorgio.moscato@uniroma1.it \\ *giampaolo.romano@uniroma1.it
}

\section{Background}

Plunging jets are used in many industrial and civil applications, as for example in sewage and water treatment plants, in order to enhance aeration and mass transfer of volatile gases. They are also observed in natural processes as rivers self-purification, waterfalls and weirs.

Many investigations dealt with the plunging jets in different configurations, but the dependence on Reynolds number and jet geometry were still not sufficiently addressed. For example, Mishra et al. (2020) studied an oblique submerged water impinging jet at different nozzle-to-plate distances and impingement angles, but only at a rather small Reynolds numbers (2600). On the other hand, different jet geometries have been extensively considered, but not for the plunging jet configuration (Mi, 2000; Hashiehbaf \& Romano, 2013).

In this work, plunging water jets issuing in air from orifices of different shape are considered. The aim of the work is to detail and compare jet behaviors in terms of velocity fields generated after impacting the air-water interface, as a function of Reynolds number and orifice geometry. However, air bubbles entrainment is mainly avoided in order to study the jet characteristics in a simpler case and use it as a reference starting point for future works.

\section{Facility and set-up}

The experimental facility is composed of a settling chamber, a wide chamber which represents the plunging pool, two discharge tanks, a centrifugal pump and an upper reservoir, as reported in Figure 1 on the left. Water plunging jets develops in air, downstream sharp-edged orifice plates of two different shapes i.e. circular and rectangular. An example of plunging jet from the rectangular orifice is reported in Figure 1 at the top right, where the axis switching phenomenon can be noted. Measurements have been performed at different Reynolds numbers in the range $10000 \div 26000$, based on the orifice diameter (equal to $2 \mathrm{~cm}$ ) and on the average exit velocity, as derived from flow rates.

The velocity field behaviors are investigated by means of Particle Image Velocimetry (PIV), using a continuous laser diode (maximum power $15 \mathrm{~W}$ ) and a high speed video-camera, FastCam Mini AX100 (up to $4000 \mathrm{frames} / \mathrm{s}$ at the maximum spatial resolution of $1024 \times 1024$ pixels). Glass microspheres of diameter in the interval $5 \div 25 \mu \mathrm{m}$ are used as tracers (Stokes time scale around $3 \times 10^{-5} \mathrm{~s}$ ).

\section{Results}

As examples of obtained results, the average velocity magnitudes are presented at the top of Figure 2 for the cases of jets issuing from circular and rectangular orifices and plunging in water, for a Reynolds number around 25000. For the circular case, the jet appears to develop in a symmetric way (close to the behavior of a free turbulent jet), having a first part characterized by the presence of the potential core till around 2 jet diameters downstream, while the jet axial velocity shows a linear decay from around 3 jet diameters. On the other hand, looking at the jet half velocity width trends, their behaviors appear to be different in the upper and lower part of the jet, suggesting a slightly different evolution of the two shear layers (but close to a symmetric condition). For the rectangular case, the jet velocity magnitude map and its vorticity field show a clear non-symmetric condition, characterized by the presence of a double-peak velocity along cross sections till a distance of about 4 diameters streamwise, from which the jet starts to have a gaussian-like distribution, as for the circular case. Moreover, the vorticity field suggests the presence of 4 shear layers 
which develop in a non-symmetric way and with a different axial velocity decay. This condition can be explained by the jet shape, which exhibits more than two collar-like meniscus forms at the plunging point.

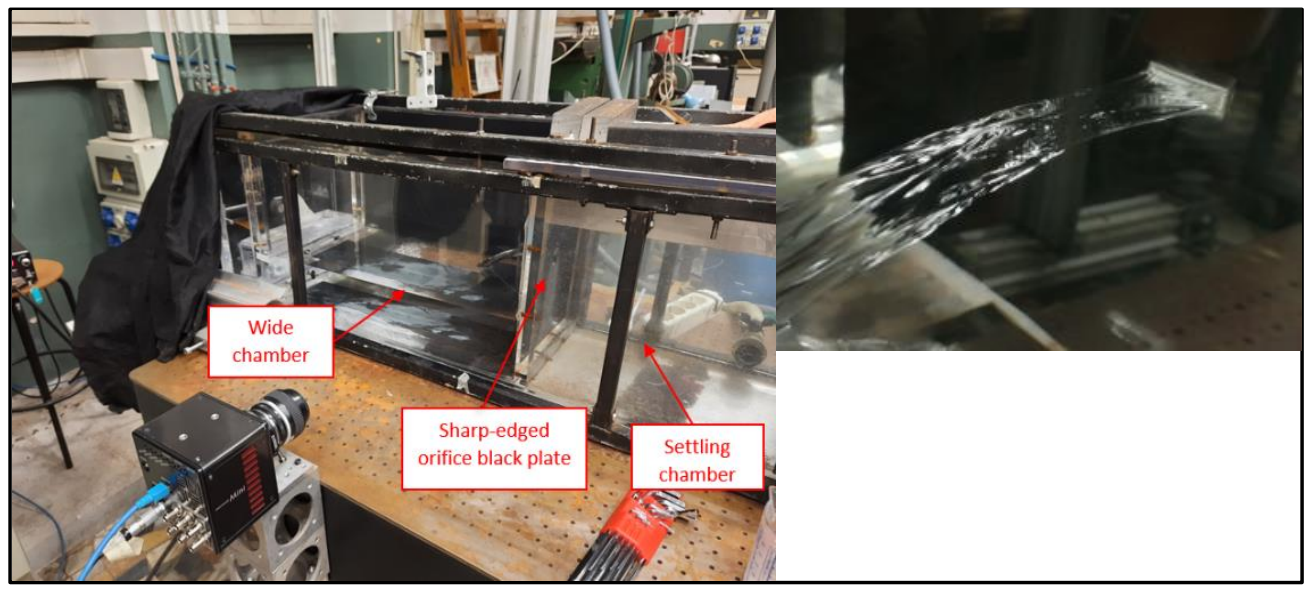

Figure 1. The facility for generation and investigation of plunging jets (on the left) and an example of plunging jet issuing from the rectangular orifice (on the right at the top).
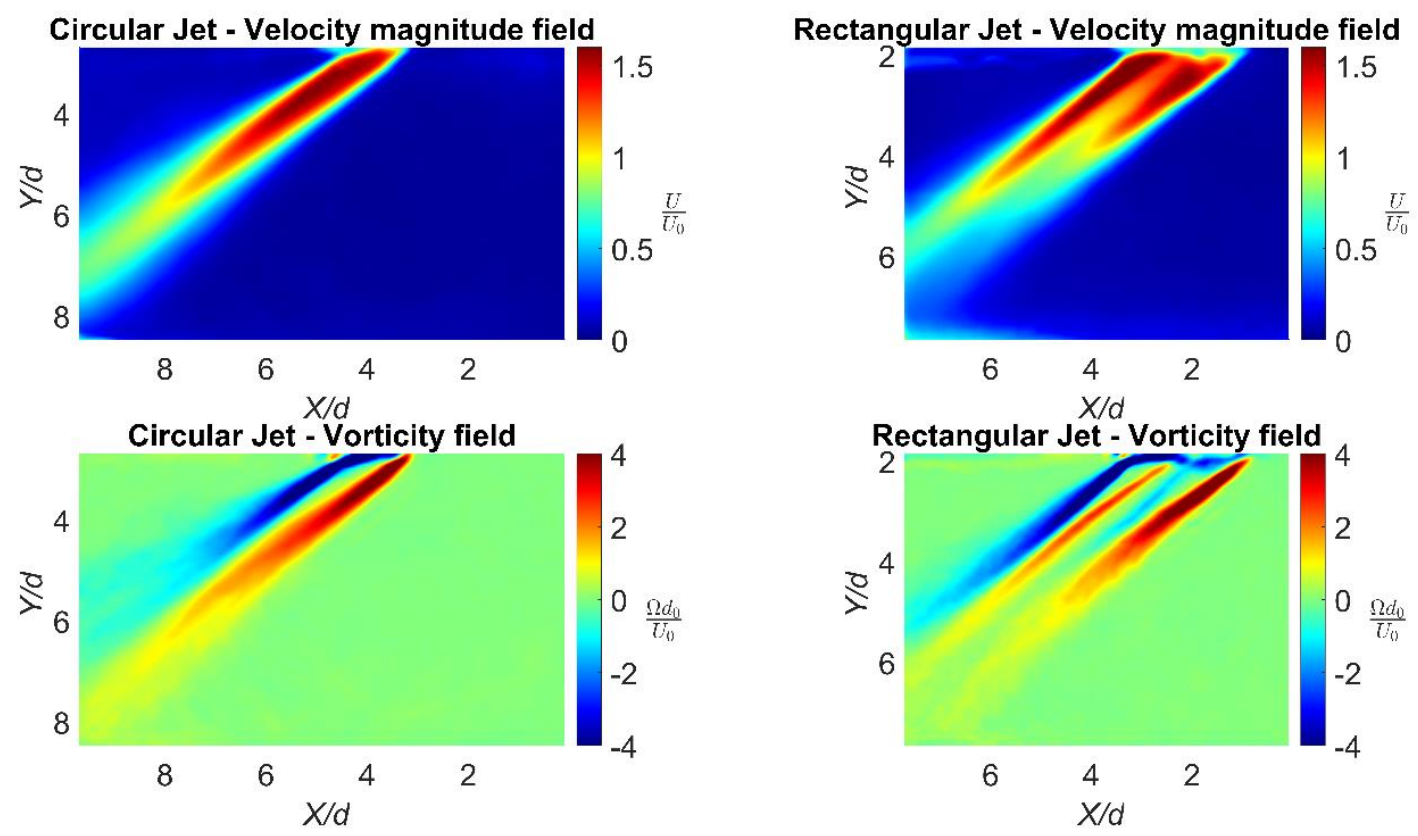

Figure 2. Mean velocity magnitude fields (at the top) and mean vorticity fields (at the bottom) for the circular and rectangular plunging jets in water as derived at $\operatorname{Re} \approx 25000$.

\section{References}

Hashiehbaf, A., \& Romano, G. (2013). Particle image velocimetry investigation on mixing enhancement of non-circular sharp edge nozzles. International Journal of Heat and Fluid Flow - Elsevier.

J. Mi, G. J. (2000). Centreline mixing characteristics of jets from nine differently shaped nozzles. Experiments in Fluids. Mishra, A., Yadav, H., Djenidi, L., \& Agrawal, A. (2020). Experimental study of fow characteristics of an oblique impinging jet. Experiments in Fluids - Springer. 